\title{
Sarcopenic obesity and cognitive performance
}

This article was published in the following Dove Press journal:

Clinical Interventions in Aging

\author{
Magdalena I Tolea' \\ Stephanie Chrisphonte \\ James E Galvin ${ }^{1,2}$ \\ 'Charles E. Schmidt College of \\ Medicine, Department of Integrated \\ Medical Sciences, Comprehensive \\ Center for Brain Health, Florida \\ Atlantic University, Boca Raton, FL, \\ USA; ${ }^{2}$ Christine E. Lynn College \\ of Nursing, Louis and Anne Green \\ Memory and Wellness Center, Florida \\ Atlantic University, Boca Raton, FL, \\ USA
}

Correspondence: Magdalena I Tolea Charles E. Schmidt College of Medicine, Department of Integrated Medical Sciences, Comprehensive Center for Brain Health, Florida Atlantic University, 777 Glades Road, ME-104, Rm I02R,

Boca Raton, FL 3343I, USA

Tel +I 56I 2974813

Fax +l 8444683794

Email mtolea@health.fau.edu
Background: Sarcopenia and obesity both negatively impact health including cognitive function. Their coexistence, however, can pose an even higher threat likely surpassing their individual effects. We assessed the relationship of sarcopenic obesity with performance on global- and subdomain-specific tests of cognition.

Patients and methods: The study was a cross-sectional analysis of data from a series of community-based aging and memory studies. The sample consisted of a total of 353 participants with an average age of 69 years with a clinic visit and valid cognitive (eg, Montreal Cognitive Assessment, animal naming), functional (eg, grip strength, chair stands), and body composition (eg, muscle mass, body mass index, percent body fat) measurements.

Results: Sarcopenic obesity was associated with the lowest performance on global cognition ${\text { (Est. }{ }_{\text {Definitionl }}=-2.85 \pm 1.38, p=0.039 \text { ), followed by sarcopenia }}^{\text {(Est. }}$ Definition1 $=-1.88 \pm 0.79, p=0.017$ ) and obesity (Est. Definition $1=-1.10 \pm 0.81, p=0.175$ ) adjusted for sociodemographic factors. The latter, however, did not differ significantly from the comparison group consisting of older adults with neither sarcopenia nor obesity. Subdomain-specific analyses revealed executive function (Est. ${ }_{\text {Definition } 1}=-1.22 \pm 0.46$ for sarcopenic obesity; Est. $_{\text {Definition } 1}=-0.76 \pm 0.26$ for sarcopenia; Est. $_{\text {Definition }}=-0.52 \pm 0.27$ for obesity all at $p<0.05$ ) and orientation (Est. ${ }_{\text {Definition } 1}=0.59 \pm 0.26$ for sarcopenic obesity; Est. ${ }_{\text {Definition1 } 1}=-0.36 \pm 0.15$ for sarcopenia; Est. ${ }_{\text {Definition } 1}=-0.29 \pm 0.15$ all but obesity significant at $p<0.05$ ) as the individual cognitive skills likely to be impacted. Potential age-specific and depression effects are discussed.

Conclusion: Sarcopenia alone and in combination with sarcopenic obesity can be used in clinical practice as indicators of probable cognitive impairment. At-risk older adults may benefit from programs addressing loss of cognitive function by maintaining/improving strength and preventing obesity.

Keywords: sarcopenia, obesity, sarcopenic obesity, cognition, cross-sectional studies

\section{Introduction}

Changes in body composition including a shift toward higher fat mass and decreased lean muscle mass $(\mathrm{MM})$ represent a significant public health concern among older adults as they may lead to various negative health outcomes including cardiovascular and neurodegenerative diseases. Higher body mass index (BMI) is inversely related to global cognition and subdomains such as executive function and processing speed. ${ }^{1,2}$ In addition, higher levels of Alzheimer's disease (AD) pathology ${ }^{3}$ and structural brain changes have been found in obese adults regardless of their cognitive status., Finally, an elevated risk of late-life dementia has been reported in middle-aged obese individuals ${ }^{6}$ suggesting a cumulative effect throughout the lifespan. ${ }^{7}$ The findings are fairly consistent across various measures of obesity. ${ }^{6,8}$ However, while obesity in early adulthood and middle age may increase risk for dementia, later in life it may not pose the same risk. ${ }^{9}$ The exact mechanisms linking obesity to cognitive dysfunction 
are yet to be determined, although several pathways including sedentary behavior, inflammation, and vascular damage have been proposed. . $^{7}$

Sarcopenia or age-related loss of MM and function may represent another, perhaps even more important, predictor of reduced cognition. In epidemiologic studies, sarcopenia has been linked to global cognitive impairment and dysfunction in specific cognitive skills including memory, speed, and executive function. ${ }^{10-13}$ National Health and Nutrition Examination Survey (NHANES) data confirm the link between sarcopenia and cognitive dysfunction in older adults and suggest inflammation as a contributing factor at least in women. ${ }^{13}$ Other proposed pathways include behavioral, ${ }^{14}$ oxidative, ${ }^{15}$ and metabolic mechanisms. ${ }^{16,17}$ In addition, reduced lean mass is associated with brain atrophy in $\mathrm{AD}^{18}$ as well as in cognitively normal older populations. ${ }^{19}$ Baseline sarcopenia predicts cognitive decline ${ }^{20}$ and development of AD and mild cognitive impairment (MCI). ${ }^{21}$ Neuropsychiatric symptoms are also more prevalent once MCI is diagnosed. ${ }^{22,23}$

Given these individual effects, presence of combined sarcopenia and obesity captured by the concept of sarcopenic obesity is likely to have an even stronger impact on cognition. This hypothesis found empirical support in a recent cross-sectional analysis of NHANES data, in which higher waist girth was linked to poor cognition, followed by low $\mathrm{MM}$ and finally by the presence of both phenotypes due in part to higher insulin resistance in the these groups compared to healthy older adults. ${ }^{24}$ In light of evidence that loss of muscle strength (MS) may have a stronger impact on health outcomes than loss of $\mathrm{MM},{ }^{25}$ the current study was designed to investigate the association between sarcopenic obesity and cognitive function using two sarcopenia definitions that incorporate measures of muscle function in addition to $\mathrm{MM}$, and also to assess this association in relation to global as well as specific aspects of cognition. Based on prior reports, we hypothesized sarcopenia alone or in combination with obesity to predict poorer performance on global- and domain-specific aspects of cognition, in particular executive function.

\section{Patients and methods}

Study participants were adults enrolled in research studies between 02/2012 and 03/2015. A detailed description of these studies has been published previously. ${ }^{11}$ Briefly, community dwelling adults aged $\geq 40$ years residing in the local catchment area (Manhattan, Queens, and Brooklyn) were recruited via collaborations with local community partners, word-of-mouth, educational seminars, and from an in-house research registry to enroll in cognitive and functional studies. The protocol, which was approved by the New York University Institutional Review Board and signed by each participant, was similar across all studies in terms of physical and cognitive assessments and other collection procedures. Exclusion criteria were age $<40$ years, nonfluency in English or Spanish, and active psychiatric and neurological conditions that could impact physical and/ or cognitive performance or could otherwise interfere with participation. A total of 353 participants with data on body composition, physical function, and cognition were included in this cross-sectional analysis. All study participants provided written informed consent.

\section{Cognition}

Performance on the Montreal Cognitive Assessment (MoCA) test was used to measure global cognitive function. MoCA can detect impairment in older adults with a sensitivity of $90 \%$ for MCI and $100 \%$ for mild $\mathrm{AD}^{26}$ and also assesses several cognitive domains commonly affected in early $\mathrm{AD}$, including short-term memory, visuospatial skills, executive function, attention and concentration, language, and orientation for a total of 30 points. Higher scores indicate better performance and scoring $<26$ can be used to define cognitive impairment in community samples. An additional point is added for those with 12 years or less of formal education to account for differences in education. In addition, scores for individual items were used to measure specific cognitive skills including visuospatial/executive function by Trail Making B, verbal abstraction, and clock drawing; language by naming, sentence repetition, and word fluency; and attention measured by finger tapping to letter, serial subtraction, and digits forward and backward. Orientation to time and place was measured by six items and short-term memory by delayed recall of five nouns.

The animal naming test, another dementia screening test, was used as a second cognitive measure of verbal fluency and mental flexibility. As part of this test, participants are asked to name as many animals as they can think of in 60 seconds. A score of $<14$ is indicative of cognitive impairment. ${ }^{27}$ Trail Making A (TMA), a measure of visual search speed, scanning, and processing speed, was also included as an additional cognitive performance measure independent of the MoCA.

\section{Sarcopenic obesity}

While the definition of sarcopenic obesity as the combination of sarcopenia and obesity is well accepted, its measurement is arbitrary due in part to the various ways the two components are measured. The definition of sarcopenia evolved overtime 
from reduced MM to later on to include reduced muscle function. Baumgartner was among the first to define sarcopenia within the former context using dual X-ray absorptiometry (DXA). ${ }^{28}$ Using similar methods to derive MM, measures that adjusted for body size followed. ${ }^{29}$ More easily accessible methods to measure $\mathrm{MM}$ in the clinical setting including bioelectrical impedance (BIA) were later proposed..$^{30}$ More recently, reduced muscle function measured as low $\mathrm{MS}^{31}$ or low MS and/or poor physical performance were added. . $^{32,33}$

Sarcopenia was measured in our study with the short portable sarcopenia measure (SPSM) proposed by Miller et al and validated for use in older populations. ${ }^{33}$ SPSM follows the current sarcopenia diagnosis guidelines and incorporates measures of MM, MS, and physical performance. Body composition was measured in our study by BIA with the BC-558 Ironman Segmental Body Composition Monitor (Tanita Corporation, Arlington Heights, IL, USA). Handgrip strength was measured by dynamometry (Baseline ${ }^{\circledR}$ Digital Smedley Spring Dynamometer; Patterson Medical, Warrenville, IL, USA) in both hands and the mean expressed as $\mathrm{kg} / \mathrm{m}$ was used in data analysis. Participants were also asked to stand five times from a seated position as fast as possible and the time in seconds was recorded. We used sex-specific cutoff points to divide participants in quintiles of lean BMI, grip strength, and chair stands. A summary score was derived using a weighted scheme in accordance to Miller et al's algorithm and sarcopenia was defined as the bottom two quintiles of this summary score. ${ }^{33}$ A second formula included low MM and/or low MS using the same cutoff. This second definition was based on the notion of strength as a measure of muscle function. However, given that muscle weakness may have a stronger negative health impact than reduced lean mass, we allowed an independent contribution of low strength to the definition of sarcopenia in our study.

Obesity has been traditionally defined as BMI $\geq 30 \mathrm{~kg} / \mathrm{m}^{2},{ }^{34}$ although other body composition measures such as fat mass, percent body fat (PBF), waist circumference (WC), and waist-to-hip ratio are often used to capture the concept of obesity using various cutoff points including sample median, ${ }^{28}$ highest two quintiles, ${ }^{35}$ or sex-specific preset values (eg, WC $>102 \mathrm{~cm}$ for men and $88 \mathrm{~cm}$ for women). ${ }^{34}$ Obesity was measured in the current study not only as BMI $\geq 30 \mathrm{~kg} / \mathrm{m}^{2}$ but also as the top two quintiles of PBF to offset inherited limitations of BMI as a weight measure.

Using these different sarcopenia and obesity definitions, we created four sarcopenic obesity indexes covering various aspects of physical function and body composition to measure results' reliability and help discern the contribution of individual components/measures (eg, overall vs visceral obesity). The first definition combines the lowest two quintiles of SPSM and BMI $\geq 30 \mathrm{~kg} / \mathrm{m}^{2}$. The second combines the lowest two quintiles of SPSM and the highest two quintiles of PBF. The third defines sarcopenic obesity as the lowest two quintiles of $\mathrm{MM}$ and $/$ or MS and BMI $\geq 30 \mathrm{~kg} / \mathrm{m}^{2}$. The last definition uses the lowest two quintiles of MM and/or MS and the highest two quintiles of PBF. For each of these different definitions, a 4 -value predictor variable was created: $0=$ no obesity no sarcopenia group (controls); $1=$ obesity group $(\mathrm{O})$; 2=sarcopenia group (S); 3=sarcopenic obesity group (SO). The control group was used as the reference group.

\section{Covariates}

Age and race (white: $68.3 \%$; black: $24.7 \%$; other: $7 \%$ ) were used as covariates. Sex (female: 65.4\%) was not included since sex-specific estimates of MM, MS, chair stands, and PBF were used to compute our predictor measure. Depressive symptomatology was measured with the Hospital Anxiety and Depression Scale (HADS) ${ }^{36}$ A cutoff of 11 was used to indicate presence of depression.

\section{Data analysis}

Differences in age- and race-adjusted MoCA means across the four classes of our predictor were analyzed using analysis of covariance. In general, generalized linear models (GLM) and total MoCA and its five subdomains were regressed on the predictor using the four different definitions adjusted for age and race. These models were run on animal naming as a second outcome measure. Also, to account for the impact of depression on physical and cognitive performance, ${ }^{37-38}$ we re-analyzed the relationships of interest controlling for depression. All analyses were performed using SAS 9.3 (SAS Institute Inc., Cary, NC, USA). A $p<0.05$ was used to evaluate statistical significance.

\section{Results}

Participants were on average 69 years old $(<8 \%$ were $<55$ years), female, and white (Table 1). Body composition, strength, and cognitive data were (all three) available in $>90 \%$ of participants. Of these, $42 \%$ had sarcopenia according to Miller's definition, whereas $62 \%$ had sarcopenia by the second definition. Using BMI $\geq 30 \mathrm{~kg} / \mathrm{m}^{2}, 32 \%$ of participants were found to be obese, whereas $42 \%$ were in the top two quintiles of PBF. Using definition 1 for our predictor yielded the most conservative SO prevalence: $14.3 \%$, while the highest prevalence $(37.2 \%)$ was found using definition 2 . 
Table I Sample characteristics according to sarcopenic obesity category (based on definition I)

\begin{tabular}{|c|c|c|c|c|}
\hline Characteristics & Controls & Obesity & Sarcopenia & $\begin{array}{l}\text { Sarcopenic } \\
\text { obesity }\end{array}$ \\
\hline Age, years & $65.87 \pm 0.89$ & $66.98 \pm 1.02$ & $73.02 \pm 0.90$ & $71.17 \pm 2.21$ \\
\hline Female, \% & $76(68.5)$ & 61 (7I.8) & $73(67.0)$ & $15(83.3)$ \\
\hline White race, \% & $66(66.0)$ & $43(54.4)$ & $73(69.5)$ & $9(50.0)$ \\
\hline BMI & $25.37 \pm 0.30$ & $35.09 \pm 0.35$ & $24.92 \pm 0.31$ & $32.16 \pm 0.76$ \\
\hline Body fat, $\%$ & $30.70 \pm 0.74$ & $41.83 \pm 0.83$ & $32.5 I \pm 0.75$ & $42.48 \pm 1.92$ \\
\hline Muscle strength, lb & $63.74 \pm 1.82$ & $56.62 \pm 2.08$ & $42.8 \mathrm{I} \pm 1.87$ & $35.23 \pm 4.53$ \\
\hline Muscle mass, lb & $98.59 \pm 2.10$ & $108.17 \pm 2.39$ & $93.97 \pm 2.15$ & $95.53 \pm 5.50$ \\
\hline MoCA & $23.86 \pm 0.50$ & $22.5 I \pm 0.58$ & $21.67 \pm 0.51$ & $20.83 \pm 1.25$ \\
\hline Charlson index & $4.89 \pm 0.19$ & $5.22 \pm 0.22$ & $5.83 \pm 0.19$ & $5.33 \pm 0.50$ \\
\hline Depression & $4.56 \pm 0.37$ & $5.90 \pm 0.43$ & $5.77 \pm 0.39$ & $5.20 \pm 1.01$ \\
\hline
\end{tabular}

Note: Bold values indicate significant difference from the sarcopenic obesity group at $p<0.05$.

Abbreviations: BMI, body mass index; MoCA, Montreal Cognitive Assessment.

In GLM models controlled for age and race, SO status was associated with MoCA in a dose-response pattern. Compared to controls, all other groups performed poorer on MoCA. However, the "risk" of poor cognitive performance was lowest in the $\mathrm{O}$ and highest in the SO, whereas the $\mathrm{S}$ group performed in the middle (Table 2). For example, while the reduction in the MoCA score had the magnitude of 1.10 in the $\mathrm{O}$ group, the effect was almost triple in the SO group (ie, -2.85) with a decrease of 1.88 units in the $\mathrm{S}$ group (Table 2; Definition 1 column). This finding was consistent across all four SO definitions investigated in this study. However, the effects are somewhat stronger when PBF rather than $\mathrm{BMI}$ is used to define obesity. Adjusted mean differences in MoCA among the four comparison groups are presented graphically in Figure 1 for the two SO definitions using BMI $\geq 30 \mathrm{~kg} / \mathrm{m}^{2}$ as evidence of obesity.

We next investigated the effect on individual cognitive subdomains. The results of the models based on the two SO definitions using BMI $\geq 30 \mathrm{~kg} / \mathrm{m}^{2}$ (ie, definitions 1 and 3) are presented in Table 3 . We found evidence of a significant negative association with executive function and orientation, where performance was poorest in $\mathrm{SO}$ (eg, Est. $=-1.22 \pm 0.46$, $p=0.009)$, followed by $\mathrm{S}($ Est. $=-0.76 \pm 0.26, p=0.005)$, and finally by $\mathrm{O}$ (Est. $=-0.52 \pm 0.27, p=0.060$ ) when compared to controls using definition 1. Definitions 2 and 4 yielded similar results (data not shown).

To determine findings' reliability, the analysis was repeated for animal naming. As can be observed in Table 4, presence of $\mathrm{SO}$ as measured by the two definitions based on PBF (ie, definitions 2 and 4) was associated with the lowest cognitive performance. While the direction of association was consistent with the MoCA findings, neither of its two components managed to reach statistical significance independently of each other. By contrast, BMI $\geq 30 \mathrm{~kg} / \mathrm{m}^{2}$ failed to distinguish between the four groups (data not shown). The SO group also showed the greatest degree of impairment across all groups when assessing TMA as an outcome. For example, using definition 2, we found the $\mathrm{SO}$ group to perform the poorest on TMA (Est. $=15.49 \pm 8.26, p=0.062$ ), followed by the $\mathrm{S}$ group $(\mathrm{Est} .=15.04 \pm 6.97, p=0.032)$ and finally by the

Table 2 Sarcopenic obesity in relation to MoCA (obesity: BMI $\geq 30 \mathrm{~kg} / \mathrm{m}^{2}$ )

\begin{tabular}{|c|c|c|c|c|c|c|c|c|}
\hline \multirow[t]{3}{*}{ Model term } & \multicolumn{4}{|c|}{ Obesity: $\mathrm{BMI} \geq 30 \mathrm{~kg} / \mathrm{m}^{2}$} & \multicolumn{4}{|c|}{ Obesity: highest two quintiles of PBF } \\
\hline & \multicolumn{2}{|l|}{ Definition I } & \multicolumn{2}{|l|}{ Definition 3} & \multicolumn{2}{|l|}{ Definition 2} & \multicolumn{2}{|l|}{ Definition 4} \\
\hline & Coefficient \pm SE & $p$-value & Coefficient \pm SE & $p$-value & Coefficient \pm SE & p-value & Coefficient \pm SE & $p$-value \\
\hline Intercept & $28.18 \pm 2.38$ & $<0.001$ & $28.0 I \pm 2.37$ & $<0.001$ & $27.52 \pm 2.37$ & $<0.001$ & $27.62 \pm 2.32$ & $<0.001$ \\
\hline Controls & 0 & - & 0 & - & 0 & - & 0 & - \\
\hline Obesity & $-1.10 \pm 0.8 \mid$ & 0.175 & $-1.02 \pm 1.02$ & 0.315 & $-1.6 I \pm 0.79$ & 0.041 & $-1.61 \pm 0.98$ & 0.103 \\
\hline Sarcopenia & $-1.88 \pm 0.79$ & 0.017 & $-1.87 \pm 0.89$ & 0.035 & $-1.80 \pm 0.83$ & 0.031 & $-1.88 \pm 0.89$ & 0.034 \\
\hline Sarcopenic obesity & $-2.85 \pm 1.38$ & 0.039 & $-2.63 \pm 1.16$ & 0.024 & $-3.14 \pm 1.00$ & 0.002 & $-3.63 \pm 1.01$ & $<0.001$ \\
\hline $\mathrm{N}$ & 295 & & 289 & & 295 & & 289 & \\
\hline
\end{tabular}

Notes: Models are adjusted for age and race; Definition I=lowest two quintiles of SPSM+BMI $\geq 30 \mathrm{~kg} / \mathrm{m}^{2}$; Definition $2=$ lowest two quintiles of SPSM+highest two quintiles of PBF; Definition 3=lowest two quintile of MM and/or MS+BMI $\geq 30$; Definition 4=lowest two quintile of MM and/or MS+highest two quintile of PBF. MS is average of right and left grip strength. Bold values represent significant differences from the control group.

Abbreviations: MoCA, Montreal Cognitive Assessment; BMI, body mass index; SE, standard error; N, number of participants; SPSM, short portable sarcopenia measure; PBF, percent body fat; MM, muscle mass; MS, muscle strength. 


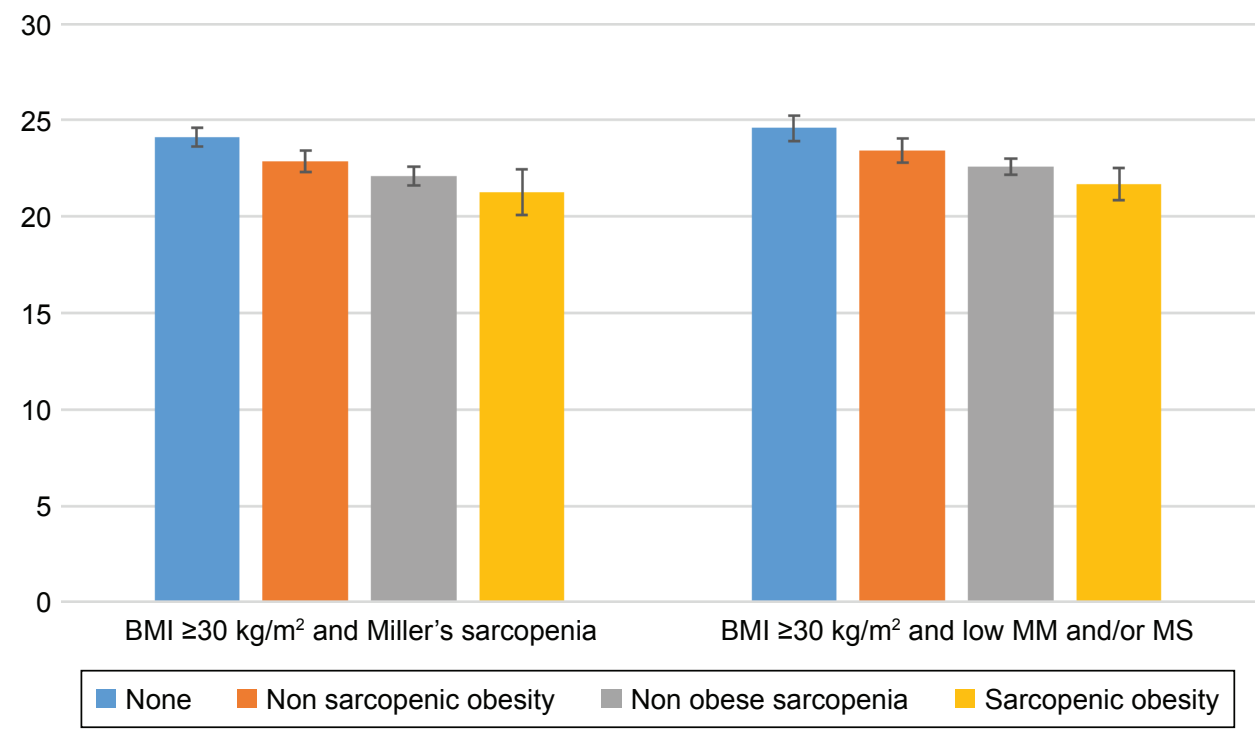

Figure I Age- and race-adjusted mean global cognition score according to sarcopenic obesity category using definitions I and 3 .

Abbreviations: BMI, body mass index; MM, muscle mass; MS, muscle strength.

O group (Est. $=14.16 \pm 6.11, p=0.021)$ compared to the control group. A longer time taken to complete TMA indicates a higher likelihood of impairment. While the SO group was only marginally different from the control group as indicated by the $p$ level of 0.062 , in analyses using $\mathrm{MM} /$ function as the sarcopenia measure of choice, the SO group performed significantly poorer than the control group (Est. $=23.32 \pm 9.38$, $p=0.014$ for definition 3; Est. $=17.33 \pm 8.35, p=0.039$ for definition 4), in line with the MoCA and animal naming findings.

Finally, the effect of our predictor was not significantly impacted when models were adjusted for depression, particularly those models predicting executive function. However, some evidence of confounding/mediation was found for MoCA (Est. ${ }_{\mathrm{o}}=-0.79 \pm 0.81, p=0.329$; Est. $._{\mathrm{s}}=-1.42 \pm 0.79$, $p=0.08$; Est. $_{\text {SO }}=-2.33 \pm 1.47, p=0.114$ for definition 1) and orientation $\left(\right.$ Est. $_{\mathrm{O}}=-0.13 \pm 0.19, p=0.515$; Est $_{\mathrm{s}}=-0.19 \pm 0.17$, $p=0.273$; Est. . $_{\mathrm{sO}}=-0.38 \pm 0.23, p=0.097$ for definition 3 ) when $\mathrm{BMI} \geq 30 \mathrm{~kg} / \mathrm{m}^{2}$ was used.

\section{Discussion}

Using a cross-sectional design, we found consistent evidence to link SO to poor global cognitive performance in community-dwelling older adults. This effect is best captured by its sarcopenia component with obesity likely having an additive effect. This effect extends to specific cognitive skills, in particular executive function and orientation. Understanding the mechanisms through which this syndrome may affect cognition is important as it may inform efforts to prevent cognitive decline in later life by targeting at-risk groups with an imbalance between lean and fat mass.

The less consistent independent effect of obesity is not surprising. Although obesity has been linked to cognitive deficits, ${ }^{2}$ structural brain changes, ${ }^{4,5}$ and $\mathrm{AD}$ pathology, ${ }^{3}$ its impact may be limited to earlier stages of adulthood, ${ }^{9}$ with inconsistent reports in samples of older adults. While some found obesity to be related to impaired cognitive function ${ }^{39}$ and cognitive decline, ${ }^{40}$ other reports showed improved

Table 3 Sarcopenic obesity in relation to individual cognitive domains (obesity: BMI $\geq 30 \mathrm{~kg} / \mathrm{m}^{2}$ )

\begin{tabular}{|c|c|c|c|c|c|c|c|c|}
\hline \multirow{2}{*}{$\begin{array}{l}\text { Cognitive } \\
\text { domains }\end{array}$} & \multicolumn{4}{|c|}{ Definition I } & \multicolumn{4}{|c|}{ Definition 3} \\
\hline & None & Obesity & Sarcopenia & $\begin{array}{l}\text { Sarcopenic } \\
\text { obesity }\end{array}$ & None & Obesity & Sarcopenia & $\begin{array}{l}\text { Sarcopenic } \\
\text { obesity }\end{array}$ \\
\hline Executive function & 0 & $-0.52 \pm 0.27$ & $-0.76 \pm 0.26$ & $-1.22 \pm 0.46$ & 0 & $-0.55 \pm 0.27$ & $-0.65 \pm 0.28$ & $-1.16 \pm 0.35$ \\
\hline Language & 0 & $-0.1 I \pm 0.20$ & $-0.38 \pm 0.19$ & $0.47 \pm 0.35$ & 0 & $0.02 \pm 0.20$ & $-0.26 \pm 0.21$ & $-0.38 \pm 0.25$ \\
\hline Attention & 0 & $-0.58 \pm 0.24$ & $-0.38 \pm 0.24$ & $-0.33 \pm 0.41$ & 0 & $-0.7 I \pm 0.23$ & $-0.26 \pm 0.25$ & $-0.83 \pm 0.30$ \\
\hline Delayed memory & 0 & $-0.11 \pm 0.27$ & $-0.30 \pm 0.26$ & $-0.68 \pm 0.46$ & 0 & $-0.49 \pm 0.26$ & $-0.47 \pm 0.28$ & $-0.74 \pm 0.33$ \\
\hline Orientation & 0 & $-0.29 \pm 0.15$ & $-0.36 \pm 0.15$ & $-0.59 \pm 0.26$ & 0 & $-0.34 \pm 0.15$ & $-0.40 \pm 0.15$ & $-0.45 \pm 0.19$ \\
\hline
\end{tabular}

Notes: Bold values indicate significant difference from the control group; models are adjusted for age and race. Abbreviation: BMI, body mass index. 
Table 4 Animal naming across sarcopenic obesity categories when obesity is defined as being in the upper two quintiles of percent body fat

\begin{tabular}{|c|c|c|c|c|}
\hline \multirow[t]{2}{*}{ Model term } & \multicolumn{2}{|l|}{ Definition 2} & \multicolumn{2}{|l|}{ Definition 4} \\
\hline & Coefficient \pm SE & $p$-value & Coefficient \pm SE & $p$-value \\
\hline Intercept & $31.72 \pm 2.79$ & $<0.001$ & $31.96 \pm 2.72$ & $<0.001$ \\
\hline Controls & 0 & - & 0 & - \\
\hline Obesity & $-1.29 \pm 0.98$ & 0.192 & $-0.75 \pm 1.27$ & 0.835 \\
\hline Sarcopenia & $-1.61 \pm 1.06$ & 0.131 & $-0.78 \pm 1.19$ & 0.511 \\
\hline Sarcopenic obesity & $-3.77 \pm 1.23$ & 0.003 & $-3.58 \pm 1.32$ & 0.007 \\
\hline $\mathrm{N}$ & 250 & & 250 & \\
\hline
\end{tabular}

Notes: Models are adjusted for age and race. Bold values represent significant differences from the control group. Abbreviation: SE, standard error.

cognitive performance ${ }^{41}$ and lower decline ${ }^{42}$ rate in obese older adults. The choice of obesity measures may at least in part explain these differential effects. ${ }^{9}$ In our study, when measured by BMI $\geq 30 \mathrm{~kg} / \mathrm{m}^{2}$, no effect of obesity independent of that of sarcopenia was detected. However, when measured using PBF, obesity was significantly associated with reduced global cognitive performance in one operationalization method (ie, definition 2) but not in the other (ie, definition 4). Also, regardless of measurement method, obesity was significantly associated with lower executive function but not with other cognitive subdomains. To further test the hypothesis that the effect of obesity may be limited to younger ages, we split our sample into age $<65$ years vs age $\geq 65$ years. Although an independent effect of obesity remained nonsignificant for both age groups, when combined with sarcopenia obesity was consistently linked to poorer cognitive performance in the younger group, while in older adults, that held for some (eg, Est. ${ }_{\mathrm{so}}=-0.95 \pm 0.39, p=0.018$ for executive function and Est. ${ }_{\mathrm{so}}=-2.71 \pm 1.13, p=0.017$ for MoCA using definition 2; similar results found for definition 4) but not all cognitive outcomes (ie, Est. ${ }_{\mathrm{sO}}=-2.39 \pm 1.58$, $p=0.131$ for MoCA and Est. ${ }_{\mathrm{SO}}=-0.89 \pm 0.62, p=0.150$ for executive function using definitions 1 and 3 ). Given these inconsistent findings in previous work as well as ours, it is possible that in older age, the effect of being overweight/ obese is offset by the strong impact of other risk factors including sarcopenia on cognition. Alternatively, maintenance of higher fat mass may be protective to the aging brain, guarding off against $\mathrm{AD} .{ }^{22}$ However, our results suggest that efforts to maintain a healthy weight should be made even in later life as obesity may enhance the detrimental effect of reduced MM and function.

The impact of sarcopenia on global cognitive performance was consistent in our study, retaining its significance whether defined based on Miller's SPSM or as low MM and/or MS. This finding is in line with previous reports, which have consistently linked sarcopenia to poor cognitive function. ${ }^{11,18,20,21}$ For example, in a case-control study comparing early $\mathrm{AD}$ patients with cognitively normal controls in terms of lean mass, cognitive performance, and brain volume, reduced lean mass was reported in the AD group and associated with poor cognitive performance and brain atrophy. ${ }^{18}$ In large epidemiologic studies of functional and cognitive aging, a protective role of high handgrip strength on cognitive health has been reported, ${ }^{20}$ slowing the rate of cognitive decline and decreasing the risk of AD. ${ }^{21}$ Studies using the newer sarcopenia diagnosis guidelines also report a strong association with cognitive impairment. ${ }^{10}$ Although an independent effect of sarcopenia was noted in relation to MoCA, we failed to find a significant effect when animal naming was investigated. It is possible that in the absence of obesity, the cognitive impact of sarcopenia is limited to global cognition and certain subdomains such as executive function and speed of processing ${ }^{12,13}$ rather than semantic memory.

Supporting the results of the one study reporting on the association between SO and cognitive functioning that we are aware of, ${ }^{24}$ we found that the risk of poor global cognition is even higher in older adults who present with low MM and strength in the context of high fat mass. This is line with dose-response associations with other negative health outcomes including all-cause mortality. ${ }^{43}$ However, we found a less consistent effect for obesity alone when sarcopenia is not present. This differential finding as it relates to global cognitive performance may stem from several differences in methodologic and analytical approaches. While we used high BMI and PBF to define obesity in our study, Levine and Crimmins used WC, which may be a better measure of visceral adiposity ${ }^{24}$ and therefore more likely to be associated with negative health outcomes than other obesity measures, particularly BMI. ${ }^{45}$ Another difference relates to the study population, our participants being slightly younger $(25 \%$ were $76+$ years), which may have diluted the effects at least for obesity. 
Further, our study contributes to the current body of knowledge by identifying specific cognitive abilities that may be impaired in individuals with evidence of SO. Executive function is reduced in obese older adults, ${ }^{9}$ and improvement in muscular function has been linked to enhancement of executive function in senior adults. ${ }^{46}$ In our study, obesity and sarcopenia were associated with lower executive function when assessed independently and even more so when they occurred together. Obesity may contribute to the risk of impaired executive function through vascular, behavioral, metabolic, and inflammatory mechanisms or can result from reduced impulse control, self-monitoring, and goal-directed behavior in individuals with impaired executive function ${ }^{47}$ with a negative effect on the ability to maintain energy balance. ${ }^{2}$ Sarcopenia, in turn, has been linked to impairments in abilities that relate to conflict resolution and selective attention. ${ }^{46}$ Other cognitive skills may be impaired in sarcopenic older adults whether or not in the presence of obesity including orientation to time and space. How and in what direction this latter association may operate should be further investigated. Impaired ability to place oneself in time and space can lead to loss of independence and therefore identifying modifiable risk factors can help this at-risk group age in place.

Several interrelated mechanisms may explain the obesitysarcopenia-cognitive dysfunction link including decreased participation in physical activity, low-grade chronic inflammation, oxidative stress, and insulin resistance, ${ }^{48}$ all of which being by-products of the aging process. Our study was not designed to investigate potential mediators of the SO-cognitive impairment association due to data unavailability, although there is empirical support for the metabolic pathways. ${ }^{24}$ These and other proposed mechanisms should be continued to be investigated in the quest of finding the most effective interventions to delay or prevent cognitive impairment, muscle impairment, and/or adiposity.

Study limitations relate to the cross-sectional design, the population studied, as well as other methodological issues. Although we observed a consistent association between sarcopenia with or without obesity in our study, due to the cross-sectional nature of our study, we were unable to investigate the direction of this association. Prospective studies will be instrumental in assessing the predictive role of SO on rate of cognitive decline and/or development of cognitive impairment and dementia in older adults. Also, our body composition measures may have led to an underestimation of obesity. For example, PBF has been found to be underestimated when using BIA as compared to DXA absorptiometry in obese children. ${ }^{49}$ However, a high correlation between these two methods of measuring body composition was reported in middle-aged adults. ${ }^{33}$ In addition, depression, which can have a negative impact on performance testing, may confound the observed effects. However, a sensitivity analysis restricted to participants with low levels of depression (eg, HADS-D < 11) yielded similar results especially when PBF was used to measure obesity (Est. $=-3.08 \pm 1.01$, $p=0.003$ and Est. $=-3.30 \pm 1.03, p=0.002 \mathrm{SO}$ vs controls when Miller's SPSM and low MM and/or strength, respectively, were used). Finally, some concern may arise from the investigation of global- and domain-specific cognitive outcomes derived from one cognitive measure (ie, MoCA). We sought to avoid this potential limitation by incorporating two cognitive measures that were independent of the MoCA: animal naming and TMA, with similar results. The screening nature of the study under investigation precluded the inclusion of a detailed neuropsychological battery to assess cognitive function. The consistency of our results that held across different SO definitions and cognitive function measures, along with the relatively large sample of a racially diverse population, and the use of established measures are positive indicators that these reported associations are not due to chance alone.

\section{Conclusion}

Sarcopenia, either alone or in the presence of obesity, can be used in clinical practice to estimate potential risk of cognitive impairment. BIA and grip strength by dynamometry can be easily administered within the time constraints of a clinic visit, and BMI is already usually collected as part of Annual Wellness visits. Further research is needed to determine whether SO is a simple correlate of cognitive performance or also has a role in the processes that lead to cognitive loss and dementia. Sarcopenic obese older adults may benefit from interventions designed to lower the risk of cognitive loss by improving/countering the age-related imbalance between MM and function.

\section{Acknowledgment}

Data collection and analysis were supported by grants awarded to JEG by the National Institutes of Health (R01 AG040211 and P30 AG008051), the Morris and Alma Schapiro Fund, and the New York State Department of Health (DOH-2011-1004010353).

\section{Author contributions}

All authors contributed toward data analysis, drafting and critically revising the paper and agree to be accountable for all aspects of the work. 


\section{Disclosure}

The authors report no conflicts of interest in this work.

\section{References}

1. Cournot M, Marquié JC, Ansiau D, et al. Relation between body mass index and cognitive function in healthy middle-aged men and women. Neurology. 2006;67(7):1208-1214.

2. Gunstad J, Paul RH, Cohen RA, Tate DF, Spitznagel MB, Gordon E. Elevated body mass index is associated with executive dysfunction in otherwise healthy adults. Compr Psychiatry. 2007;48(1): 57-61.

3. Mrak RE. Alzheimer-type neuropathological changes in morbidly obese elderly individuals. Clin Neuropathol. 2009;28(1):40-45.

4. Ho AJ, Raji CA, Becker JT, et al; Cardiovascular Health Study; ADNI. Obesity is linked with lower brain volume in $700 \mathrm{AD}$ and MCI patients. Neurobiol Aging. 2010;31(8):1326-1339.

5. Raji CA, Ho AJ, Parikshak NN, et al. Brain structure and obesity. Hum Brain Mapp. 2010;31(3):353-364.

6. Whitmer RA, Gunderson EP, Quesenberry CP Jr, Zhou J, Yaffe K. Body mass index in midlife and risk of Alzheimer disease and vascular dementia. Curr Alzheimer Res. 2007;4(2):103-109.

7. Miller AA, Spencer SJ. Obesity and neuroinflammation: a pathway to cognitive impairment. Brain Behav Immun. 2014;42:10-21.

8. Whitmer RA, Gustafson DR, Barrett-Connor E, Haan MN, Gunderson EP, Yaffe K. Central obesity and increased risk of dementia more than three decades later. Neurology. 2008;71(14):1057-1064.

9. Smith E, Hay P, Campbell L, Trollor JN. A review of the association between obesity and cognitive function across the lifespan: implications for novel approaches to prevention and treatment. Obes Rev. 2011;12(9):740-755.

10. Hsu YH, Liang CK, Chou MY, et al. Association of cognitive impairment, depressive symptoms and sarcopenia among healthy older men in the veterans retirement community in southern Taiwan: a cross-sectional study. Geriatr Gerontol Int. 2014;14 (Suppl 1):102-108.

11. Tolea MI, Galvin JE. Sarcopenia and impairment in cognitive and physical performance. Clin Interv Aging. 2015;10:663-671.

12. Scherder EJ, Eggermont LH, Geuze RH, Vis J, Verkerke GJ. Quadriceps strength and executive functions in older women. Am J Phys Med Rehabil. 2010;89(6):458-463.

13. Canon ME, Crimmins EM. Sex differences in the association between muscle quality, inflammatory markers, and cognitive decline. J Nutr Health Aging. 2011;15(8):695-698.

14. Roubenoff R. Exercise, sarcopenia, cognition, and mood. Nestle Nutr Workshop Ser Clin Perform Programme. 2002;6:151-159; discussion $160-162$.

15. Berr C, Balansard B, Arnaud J, Roussel AM, Alpérovitch A. Cognitive decline is associated with systemic oxidative stress: the EVA study. Etude du Vieillissement Artériel. J Am Geriatr Soc. 2000;48(10): 1285-1291.

16. Dimitriadis G, Mitrou P, Lambadiari V, Maratou E, Raptis SA. Insulin effects in muscle and adipose tissue. Diabetes Res Clin Pract. 2011;93 (Suppl 1):S52-S59.

17. Geroldi C, Frisoni GB, Paolisso G, et al. Insulin resistance in cognitive impairment: the InCHIANTI study. Arch Neurol. 2005;62(7): 1067-1072.

18. Burns JM, Johnson DK, Watts A, Swerdlow RH, Brooks WM. Reduced lean mass in early Alzheimer disease and its association with brain atrophy. Arch Neurol. 2010;67(4):428-433.

19. Kilgour AH, Ferguson KJ, Gray CD, et al. Neck muscle cross-sectional area, brain volume and cognition in healthy older men: a cohort study. BMC Geriatr. 2013;13:20.

20. Alfaro-Acha A, Al Snih S, Raji MA, Kuo YF, Markides KS, Ottenbacher KJ. Handgrip strength and cognitive decline in older Mexican Americans. J Gerontol A Biol Sci Med Sci. 2006;61(8):859-865.
21. Boyle PA, Buchman AS, Wilson RS, Leurgans SE, Bennett DA. Association of muscle strength with the risk of Alzheimer disease and the rate of cognitive decline in community-dwelling older persons. Arch Neurol. 2009;66(11):1339-1344.

22. Johnson DK, Wilkins CH, Morris JC. Accelerated weight loss may precede diagnosis in Alzheimer disease. Arch Neurol. 2006;63(9):1312-1317.

23. Sanderlin AH, Alsibai A, Bozoki A. The effect of obesity on severity of cognitive impairment and neuropsychiatric symptoms in MCI subjects (I12-4B). Neurology. 2015;84(14 Suppl).

24. Levine ME, Crimmins EM. Sarcopenic obesity and cognitive functioning: the mediating roles of insulin resistance and inflammation? Curr Gerontol Geriatr Res. 2012;2012:826398.

25. Newman AB, Kupelian V, Visser M, et al. Strength, but not muscle mass, is associated with mortality in the health, aging and body composition study cohort. J Gerontol A Biol Sci Med Sci. 2006;61(1):72-77.

26. Nasreddine ZS, Phillips NA, Bédirian V, et al. The Montreal Cognitive Assessment, MoCA: a brief screening tool for mild cognitive impairment. J Am Geriatr Soc. 2005;53(4):695-699.

27. Sager MA, Hermann BP, La Rue A, Woodard JL. Screening for dementia in community-based memory clinics. WMJ. 2006;105(7):25-29.

28. Baumgartner RN. Body composition in healthy aging. Ann N Y Acad Sci. 2000;904:437-448.

29. Newman AB, Kupelian V, Visser M, et al; Health ABC Study Investigators. Sarcopenia: alternative definitions and associations with lower extremity function. J Am Geriatr Soc. 2003;51(11):1602-1609.

30. Janssen I, Heymsfield SB, Ross R. Low relative skeletal muscle mass (sarcopenia) in older persons is associated with functional impairment and physical disability. J Am Geriatr Soc. 2002;50(5):889-896.

31. Stenholm S, Harris TB, Rantanen T, Visser M, Kritchevsky SB, Ferrucci L. Sarcopenic obesity: definition, cause and consequences. Curr Opin Clin Nutr Metab Care. 2008;11(6):693-700.

32. Cruz-Jentoft AJ, Baeyens JP, Bauer JM, et al; European Working Group on Sarcopenia in Older People. Sarcopenia: European consensus on definition and diagnosis: Report of the European Working Group on Sarcopenia in Older People. Age Ageing. 2010;39(4):412-423.

33. Miller DK, Malmstrom TK, Andresen EM, et al. Development and validation of a short portable sarcopenia measure in the African American health project. J Gerontol A Biol Sci Med Sci. 2009;64(3): 388-394.

34. World Health Organization. Obesity: preventing and managing the global epidemic. Report of a WHO Consultation. (WHO Technical Report Series 894). Available from: http:/www.who.int/nutrition/ publications/obesity/WHO_TRS_894/en/. Published 2000. Accessed February 2, 2018.

35. Davison KK, Ford ES, Cogswell ME, Dietz WH. Percentage of body fat and body mass index are associated with mobility limitations in people aged 70 and older from NHANES III. J Am Geriatr Soc. 2002;50(11):1802-1809.

36. Zigmond AS, Snaith RP. The hospital anxiety and depression scale. Acta Psychiatr Scand. 1983;67(6):361-370.

37. Yanagita M, Willcox BJ, Masaki KH, et al. Disability and depression: investigating a complex relation using physical performance measures. Am J Geriatr Psychiatry. 2006;14(12):1060-1068.

38. Fitzgerald PB, Srithiran A, Benitez J, et al. An fMRI study of prefrontal brain activation during multiple tasks in patients with major depressive disorder. Hum Brain Mapp. 2008;29(4):490-501.

39. Kilander L, Nyman H, Boberg M, Lithell H. Cognitive function, vascular risk factors and education. A cross-sectional study based on a cohort of 70-year-old men. J Intern Med. 1997;242(4):313-321.

40. Elias MF, Elias PK, Sullivan LM, Wolf PA, D’Agostino RB. Obesity, diabetes and cognitive deficit: the Framingham Heart Study. Neurobiol Aging. 2005;26(Suppl 1):11-16.

41. Kuo HK, Jones RN, Milberg WP, et al. Cognitive function in normalweight, overweight, and obese older adults: an analysis of the Advanced Cognitive Training for Independent and Vital Elderly cohort. J Am Geriatr Soc. 2006;54(1):97-103. 
42. Sturman MT, de Leon CF, Bienias JL, Morris MC, Wilson RS, Evans DA. Body mass index and cognitive decline in a biracial community population. Neurology. 2008;70(5):360-367.

43. Atkins JL, Whincup PH, Morris RW, Lennon LT, Papacosta O, Wannamethee SG. Sarcopenic obesity and risk of cardiovascular disease and mortality: a population-based cohort study of older men. J Am Geriatr Soc. 2014;62(2):253-260.

44. Lemieux S, Prud'homme D, Bouchard C, Tremblay A, Després JP. A single threshold value of waist girth identifies normal-weight and overweight subjects with excess visceral adipose tissue. Am JClin Nutr. 1996;64(5):685-693.

45. Klein S, Allison DB, Heymsfield SB, et al; Association for Weight Management and Obesity Prevention; NAASO, The Obesity Society; American Society for Nutrition; American Diabetes Association. Waist circumference and cardiometabolic risk: a consensus statement from shaping America's health: Association for Weight Management and Obesity Prevention; NAASO, the Obesity Society; the American Society for Nutrition; and the American Diabetes Association. Am J Clin Nutr. 2007;85(5):1197-1202.
46. Liu-Ambrose T, Nagamatsu LS, GrafP, Beattie BL, Ashe MC, Handy TC. Resistance training and executive functions: a 12-month randomized controlled trial. Arch Intern Med. 2010;170(2):170-178.

47. Lezak MD, Howieson DB, Bigler ED, Tranel D. Neuropsychological Assessment. 5th ed. New York: Oxford University Press; 2012.

48. Zamboni M, Mazzali G, Fantin F, Rossi A, Di Francesco V. Sarcopenic obesity: a new category of obesity in the elderly. Nutr Metab Cardiovasc Dis. 2008;18(5):388-395.

49. Eisenkölbl J, Kartasurya M, Widhalm K. Underestimation of percentage fat mass measured by bioelectrical impedance analysis compared to dual energy X-ray absorptiometry method in obese children. Eur J Clin Nutr. 2001;55(6):423-429.
Clinical Interventions in Aging

\section{Publish your work in this journal}

Clinical Interventions in Aging is an international, peer-reviewed journal focusing on evidence-based reports on the value or lack thereof of treatments intended to prevent or delay the onset of maladaptive correlates of aging in human beings. This journal is indexed on PubMed Central, MedLine,

\section{Dovepress}

CAS, Scopus and the Elsevier Bibliographic databases. The manuscript management system is completely online and includes a very quick and fair peer-review system, which is all easy to use. Visit http://www.dovepress. $\mathrm{com} /$ testimonials.php to read real quotes from published authors. 